\title{
Association of lymph node promotion activity to cervical lymph node yield of oral cancer patients in Asian country: A difference-in-differences analysis
}

\author{
Ching-Chieh Yang ${ }^{1}$, Bor-Hwang Kang ${ }^{2}$, Wen-Shan Liu ${ }^{3}$, Chun-Hao Yin ${ }^{4}$, and Ching-Chih \\ Lee $^{2}$ \\ ${ }^{1}$ Chi-Mei Medical Center \\ ${ }^{2}$ Kaohsiung Veterans General Hospital \\ ${ }^{3}$ Kaohsiung Veteran General Hospital \\ ${ }^{4}$ Department of Medical Education and Research, Kaohsiung Veterans General Hospital, \\ Kaohsiung, Taiwan
}

May 6, 2020

\begin{abstract}
Objectives: High quality of lymph node yield could increase survival but strategies to improve it was seldom reported. This study was aim to assess the impact of a lymph node promotion activi-ty (LNPA) in oral cancer surgery through a difference-indifferences research design. Design: A retrospective based study. Setting: Medical center in Taiwan. Subjects and methods: A total of 400 oral cancer patients underwent primary tumor resection and neck dissection including elective neck dissection (END) and radical neck dissection (RND) were recruited after propensity score matching by clinical T and N category between 2009 Janu-ary and 2018 October. Oral cancer patients were treated by two independent departments in our institute. Since 2015 Oct, a LNPA was initiated in one department (target group), and another department was as a control group. The impact of LNPA on LN yield and regional recurrence were analyzed and compared between these two groups by difference-in-differences (DID) linear regression analysis. Results: The mean age was $55.2+11.1$ years, and $92 \%$ were male. 180 (45\%) oral cancer patients had T3-4 disease, and 128 (32\%) patients had N2-3 disease. In DID analysis, a lower LN yield among the target group before 2015 Oct (coefficient=-11.05, $\mathrm{p}<0.001$ ). LNPA launched after 2015 Oct in target group showed a positive promotion of LN yield (coefficient $=13.24, \mathrm{p}<0.001$ ). Moreover, a borderline protective effect of LNPA on regional recurrence among those with $\mathrm{cN0}$ disease (coefficient $=-9.4 \%, \mathrm{p}=0.087$ ) was noted. Conclusion: Strategy, like LNPA was associated increased LN yield and decreased regional re-currence in oral cancer. This kind of activity to promote surgeons to improve their quality of neck dissection was feasible and could be applied widespread.
\end{abstract}

\section{INTRODUCTION}

Oral cancer remains one of the most common and lethal tumors worldwide. ${ }^{1}$ Despite great advances of multimodal therapy, the treatments for oral cancer is still tough for its high recurrence and metastatic rates. $^{2}$ Currently, wide resection of the primary tumor with neck dissection is the cornerstone of treatment for patients with neck metastasis or a high occult neck metastasis rate. ${ }^{3}$ Because oral cancer patients may have a worse prognosis when recurrence occur, it is important to perform wide resection of the primary tumor with a clear margin. ${ }^{4}$ Recently, a high quality neck dissection with adequate retrieval of lymph node $(\mathrm{LN})$ was introduced and used to improve outcomes in different tumor types especially for oral cancer. ${ }^{5,6}$

Patients with inadequate lymph node harvests might experience stage migration and subsequent underestimation of disease severity. ${ }^{7}$ Previous literatures have outlined the positive association between LN yield in 
neck dissection and overall survival rates. ${ }^{8,9}$ Ebrahimi, A et al reported that LN yield $<18$ was associated with worse 5-year overall (hazard ratio [HR], 2.0; 95\% confidence interval [CI], 1.1-3.6), disease-specific (HR, 2.2; 95\% CI, 1.1-4.5), and disease-free survival (HR, 1.7; 95\% CI, 1.1-2.8). Thus, LN yield $>18$ was proposed as a cut-off point for adequate neck dissection. A cut-off value of 15 for elective neck dissection and 25 for radical neck dissection were also recommended by Lee et al. ${ }^{10}$ However, there is no efficient intervention style for neck dissection quality improvement.

Given this background, we tried to explore the change of LN yield of neck dissection among two different departments at different times in our institute. In order to improve the quality of LN yield for oral cancer patient receiving neck dissection, a lymph node promotion activity (LNPA) concerning neck dissection quality for surgeons was implemented in one department (target group) in our institute since 2015 Oct. The activity included promotion the importance of number of lymph node yield on oral cancer survival rates, identification of important landmarks during neck dissection and announce of average of cervical LN yield to surgeons per month. Oral cancer patients treated in another department without LNPA were regarded as controls. The changes of LN yield and regional recurrence by diagnosed date in different periods (before and after 2015 Oct) among target group and control group were compared with difference-in-differences analysis. ${ }^{11,12}$ Although randomized controlled trial was the best way to avoid selection bias, information bias and confounding variables, they were not always feasible in daily practice. Using a quasi-experimental study that compared outcomes of groups with exposure to different policies or interventions at different periods, the causal inference could be possible. ${ }^{13}$ If the intervention, like LNPA, has a positive impact on LN yield and reduced regional recurrence, quality improvement in oral cancer surgery could be applied and introduced widespread.

\section{MATERIALS AND METHODS}

\section{Patient demographics and database}

Newly diagnosed oral cancer patients treated with resection of the primary oral tumor and neck dissection with or without adjuvant therapy were identified from our hospital Cancer Registry Database within the years 2009 to 2018. Patients without complete treatment, electric medical records, regular follow up, and distant metastasis on diagnosis were excluded in our study. The type of neck dissection included elective neck dissection (END) for clinical N0 disease and radical neck dissection (RND) for clinical N1-3 disease. Patients with bilateral neck dissection and those with retrieved LN less than 10 were also excluded. The variables collected from the Cancer Registry Database included patient demographic data, such as age, gender, tumor status such as clinico-pathological TNM stage, and pathological risk features (such as margin status, tumor differentiation, perineural invasion, lympho-vascular permeation, number of retrieved LNs, number of positive LNs, extranodal extension, adjuvant treatment modality, radiation dose, and chemotherapy regimen). All staging was done according to the American Joint Committee on Cancer (AJCC) cancer staging (7th edition). As shown in figure 1, the study periods were categorized into two stages: period 1 (before 2015 Oct), and period 2 (after 2015 Oct). In the period 1, the operative procedure of primary tumor resection and neck dissection was performed as per the guidelines or literatures in target group and control group. ${ }^{3,14}$ Since period 2, soft but non-mandatory intervention, lymph node promotion activiy (LNPA) for cervical lymph node improvement was implemented in target group alone. The LNPA used in the target group including: (1) promotion of the importance of survival rates and LN yield in head and neck cancer in weekly meetings, and resident journal club conferences; (2) surgeons were encouraged to identify important landmarks, like cervical ansa, internal jugular vein, carotid artery, spinal accessory nerve, splenius capitus, levator scapula, anterior scalene muscle, and transverse cervical artery during neck dissection ; (3) the average number of LN yield was announced per month, but individual data related to the patients or surgeons were not disclosed (Figure 1).

The hypothesis was that target group surgeons' behavior response to the LNPA could improve LN yield in the period 2. The effect of LNPA on lymph node yield and regional recurrence was evaluated by differencein-differences analysis. For regional recurrence, we used a concept of regional recurrence density which meant the proportion of regional recurrence among those treated with neck dissection during a specific period. This 
method could provide us a comparison of regional recurrence between the target group and control groups in different periods.

\section{Statistical analysis}

The distributions of patient, tumor, and treatment characteristics were compared. Categorical variables were analyzed with the Pearson chi-square or Fisher exact test, and continuous variables were compared by one-way analysis of variance. Difference-in-differences analysis for evaluating a policy change that apply to some but not all members was used in this series. ${ }^{11,12}$ In period 1 , there was no specific intervention or policy difference for oral cancer treatment in target group and control group. In period 2, LNPA was applied for target group alone. The difference of lymph node yield between different periods (period 1 and 2), and different departments (target group and control group) was analyzed. Among difference-in-differences method, the effects of the target group (target group in period 1), the time period (period 2), and target group in the post-intervention period (target group in period 2) was analyzed through liner model for LN yield and regional recurrence. ${ }^{13}$ In order to reach the requirement of strict exogeneity, propensity score matching with clinical tumor category and node category was performed. ${ }^{15} \mathrm{~A}$ two-side test with a $\mathrm{P}$ value of $<0.05$ was set as representing statistical significance.

\section{RESULTS}

Between 2009 and 2018, a total of 469 newly diagnosed oral cancer patients receiving wide resection and neck dissection were recruited. After propensity score matching, 400 oral cancer patients were enrolled for our analysis. The mean age was $55.2+11.1$ years and $92 \%$ were male (Table 1 ). In period 1 , patients treated by target group surgeons were more likely to be older, had tongue cancer, and received adjuvant radiotherapy. In period after 2015 Oct, patients treated by target group surgeons were more likely to have tongue cancer.

The average LN yield per half-year by different departments was illustrated in Figure 2. As shown in Table 2, compared with control group in period 1, the average LN yield was lower in target group (22.8 vs 33.4, $p<0.001)$. The average LN yield was also lower in target group between different neck dissection types (21.2 vs 29.3, $\mathrm{p}<0.001$ in END group; 24.3 vs $38, p<0.001$ in RND group). However, compared with control in period 2, the average LN yield was similar between the target group and control group (37.4 vs 33.9, $p$ $=0.099) ; 34.2$ vs $33.5(\mathrm{p}=0.7)$ in END group and 41.5 vs $35(\mathrm{p}=0.11)$ in RND group. This finding means there was significant increase of LN yield in target after 2015 Oct, but no statistical difference of LN yield in control group. The difference-in-difference analysis through linear model estimated the impact of LNPA on LN yield. In Table 3, the interaction item, target group $\times$ period 2, with a coefficient of $13.24(\mathrm{p}<0.001)$ represented the improvement of LN yield in target group after launching LNPA since 2015 Oct.

We further analyzed the regional recurrence by diagnosis month. The regional recurrence rate between target and control groups was presented in Table 4 and Figure 3. During period before 2015 Oct, regional recurrence was a little higher among patients treated in target group ( $8.5 \% \mathrm{vs} 5.9 \%, \mathrm{p}=0.45)$. In period after 2015 Oct, regional recurrence in target group was a little lower than control group $(2.5 \%$ vs $3.7 \%, \mathrm{p}=0.65)$. Difference-in-differences analysis in Table 5 showed a borderline protective effect of LNPA for cN0 patients with a reduced regional recurrence rate of $9.4 \%(\mathrm{p}=0.087)$.

\section{DISCUSSION}

Although there were several breakthrough innovations in treatment, such as target therapy, or immune checkpoint inhibitors, oral cancer patients with regional recurrence incurred grave outcomes. ${ }^{2,4}$ Besides radical resection of primary tumor with adequate margin, the next step was to perform an adequate neck dissection. Previous studies had proved the positive association between LN yield and survival rates. ${ }^{8,16}$ However, strategies to improve LN yield was not clearly found in literatures. This study was the first to explore the impact of LNPA among surgeons for quality of neck dissection. Through continued education, promotion of importance of LN yield in neck dissection surgery, identification of landmarks during surgery and public announce of LN yield average to surgeons might promote the quality of neck dissection and reduce regional recurrence in turn through a difference-in-differences analysis. This soft and non-mandatory method could 
be applied to all head and neck surgeons widespread.

This study had several strengths. Using a difference-in-differences analysis, the impact of policy or intervention change that was implemented to some groups could be evaluated. ${ }^{13}$ In this study, oral cancer patients were treated by two departments in our institute, and LNPA was launched among target group in period after 2015 Oct. This situation provided us an opportunity to evaluate the possible positive effect of LNPA on LN yield and regional recurrence through a difference-in-difference analysis. Our finding of this study provided evidences to support the positive effect of LNPA. In difference-in-difference analysis, the coefficient for target group in period after 2015 Oct was $13.4(\mathrm{p}<0.001)$. In regional recurrence analysis, patients with $\mathrm{cN} 0$ disease might get borderline benefit from LNPA with a reduction of regional recurrence rate of $9.4 \%$ $(\mathrm{p}=0.087)$.

Although the study design of difference-in-differences analysis provided us a chance to explore the effect of policy or intervention at different times, several assumptions should be met. The most common assumptions were the common trends assumption and strict exogeneity. The common trend assumption meant that the unmeasured variables were time-invariant group character or time-varying factors with group invariant attributes. ${ }^{13}$ It could be examined by checking the time-series graph with a set of parallel lines. Supplementary Figure illustrated the changes of LN yield in different groups and periods. The lines looked like parallel lines. Using matching techniques that could eliminate possible selection bias between different groups and time periods might help the difference-in-difference study, like the LNPA, to reach the requirement of strict exogeneity. ${ }^{15}$ Other matching method, such as refined covariate balance could also be used. ${ }^{17}$

All national and international guidelines reported adequate LN yield could significantly improve survival in oral cancer patients, but there is no recommend intervention in clinical practice. Recently, interventions based on the theories of behavior economics are summarized by the acronym NUDGE and has been explored in the healthcare field. ${ }^{18-20}$ Penn Medicine, for example, has used this strategy widely. Changing the default in the choice of medicine greatly increased the rate of prescriptions for generic medicine. ${ }^{19}$ Using an active choice alert system in the electric medical record increased the influenza vaccination rate $37 \%$ in adults suitable for vaccination. ${ }^{21}$ Ayala et al. also reported that moving from an "opt-in" to an "opt-out" system greatly increased the rate of providing aspirin prophylaxis for preeclampsia prevention. ${ }^{22}$ Automated dashboard with active choice and peer comparison performance feedback to physicians had increased statin prescribing for primary care physicians. ${ }^{23}$ In our institute, several feasible and low-cost strategies were applied. At first, weekly conferences were used to explain the association between LN yield and recurrence since 2015 Oct. Thereafter, we encouraged the surgeons to confirm important landmarks during neck dissections came. Furthermore, the average number of LN yield was announced per month in order to give feedback to the surgeons. Multiple NUDGE-like interventions helped the target group to improve the cervical LN yield in oral cancer surgery.

There are several limitations in this study. Although we introduced several interventions to improve the quality of neck dissection in our institution, the study was not completely prospective designed. Second, we didn't perform the survival analysis due to short-term of follow-up period in those treated in period after 2015 Oct. The minimal follow-up period in our series was 18 months, which represented the least follow-up period for monitoring recurrence, and most of the recurrences could be captured. Furthermore, we used the concept of regional recurrence density, which meant the proportion of regional recurrence among those with neck dissection during a specific period. Figure 3 illustrated the change of regional recurrence density among the target group and control group. Through case matching with propensity score by clinical tumor and node category, the comparison between the two groups were more reasonable. Third, the spillover effects of LNPA to the control group was not estimated. Although it was possible, the effect might be minimal because these two departments located in different floors in our institute.

\section{CONCLUSION}

Previous literature has validated the importance of LN yield in head and neck cancer. The interventions, like LNPA, may efficiently increase the number of cervical LNs and could reduce the subsequent recurrence rate 
in oral cancer patients underwent neck dissection. Soft and non-mandatory intervention could be applied widespread in order to improve oral cancer treatment quality and survival rates.

\section{References}

1. Siegel RL, Miller KD, Jemal A. Cancer statistics, 2019. CA Cancer J Clin. 2019;69(1):7-34.

2. Rogers SN, Brown JS, Woolgar JA, et al. Survival following primary surgery for oral cancer. Oral Oncol. 2009;45(3):201-211.

3. Ettinger KS, Ganry L, Fernandes RP. Oral Cavity Cancer. Oral Maxillofac Surg Clin North Am. 2019;31(1):13-29.

4. Woolgar JA, Rogers S, West CR, Errington RD, Brown JS, Vaughan ED. Survival and patterns of recurrence in 200 oral cancer patients treated by radical surgery and neck dissection. Oral Oncol.1999;35(3):257265.

5. Lykke J, Rosenberg J, Jess P, Roikjaer O, Danish Colorectal Cancer G. Lymph node yield and tumour subsite are associated with survival in stage I-III colon cancer: results from a national cohort study. World $J$ Surg Oncol. 2019;17(1):62.

6. Visser E, Markar SR, Ruurda JP, Hanna GB, van Hillegersberg R. Prognostic Value of Lymph Node Yield on Overall Survival in Esophageal Cancer Patients: A Systematic Review and Meta-analysis. Ann Surg.2019;269(2):261-268.

7. Lee CC, Lin YS, Kang BH, et al. Incorporation of log odds of positive lymph nodes into the AJCC TNM classification improves prediction of survival in oral cancer. Clin Otolaryngol. 2017;42(2):425-432.

8. Divi V, Chen MM, Nussenbaum B, et al. Lymph Node Count From Neck Dissection Predicts Mortality in Head and Neck Cancer. J Clin Oncol. 2016;34(32):3892-3897.

9. Ebrahimi A, Clark JR, Zhang WJ, et al. Lymph node ratio as an independent prognostic factor in oral squamous cell carcinoma.Head Neck. 2011;33(9):1245-1251.

10. Lee CC, Su YC, Hung SK, et al. Recommendation for incorporation of a different lymph node scoring system in future AJCC N category for oral cancer. Sci Rep. 2017;7(1):14117.

11. Raifman J, Moscoe E, Austin SB, Hatzenbuehler ML, Galea S. Association of State Laws Permitting Denial of Services to Same-Sex Couples With Mental Distress in Sexual Minority Adults: A Difference-inDifference-in-Differences Analysis. JAMA Psychiatry. 2018;75(7):671-677.

12. Carey K, Lin MY. Readmissions To New York Hospitals Fell For Three Target Conditions From 2008 To 2012, Consistent With Medicare Goals.Health Aff (Millwood). 2015;34(6):978-985.

13. Wing C, Simon K, Bello-Gomez RA. Designing Difference in Difference Studies: Best Practices for Public Health Policy Research. Annu Rev Public Health. 2018;39:453-469.

14. Fernandes R, Ord R. Access surgery for oral cancer. Oral Maxillofac Surg Clin North Am. 2006;18(4):565571.

15. Obermeyer Z, Makar M, Abujaber S, Dominici F, Block S, Cutler DM. Association between the Medicare hospice benefit and health care utilization and costs for patients with poor-prognosis cancer.JAMA. 2014;312(18):1888-1896.

16. Cramer JD, Speedy SE, Ferris RL, Rademaker AW, Patel UA, Samant S. National evaluation of multidisciplinary quality metrics for head and neck cancer. Cancer. 2017;123(22):4372-4381.

17. Pimentel SD, Kelz RR, Silber JH, Rosenbaum PR. Large, Sparse Optimal Matching with Refined Covariate Balance in an Observational Study of the Health Outcomes Produced by New Surgeons. J Am Stat Assoc.2015;110(510):515-527. 
18. Binns C, Low WY. Nobel Prizes, Nudge Theory, and Public Health.Asia Pac J Public Health. 2017;29(8):632-634.

19. Bourdeaux CP, Davies KJ, Thomas MJ, Bewley JS, Gould TH. Using 'nudge' principles for order set design: a before and after evaluation of an electronic prescribing template in critical care. BMJ Qual Saf. 2014;23(5):382-388.

20. O'Reilly-Shah VN, Easton GS, Jabaley CS, Lynde GC. Variable effectiveness of stepwise implementation of nudge-type interventions to improve provider compliance with intraoperative low tidal volume ventilation. BMJ Qual Saf. 2018;27(12):1008-1018.

21. Patel MS, Volpp KG, Small DS, et al. Using Active Choice Within the Electronic Health Record to Increase Influenza Vaccination Rates.J Gen Intern Med. 2017;32(7):790-795.

22. Ayala NK, Rouse DJ. A Nudge Toward Universal Aspirin for Preeclampsia Prevention. Obstet Gynecol. 2019;133(4):725-728.

23. Patel MS, Kurtzman GW, Kannan S, et al. Effect of an Automated Patient Dashboard Using Active Choice and Peer Comparison Performance Feedback to Physicians on Statin Prescribing: The PRESCRIBE Cluster Randomized Clinical Trial. JAMA Netw Open. 2018;1(3):e180818.

\section{Figure legends}

Figure 1. Strategies applied to neck dissection for oral cancer in different periods ( before and after October, 2015).

Figure 2. Mean lymph node yield between target and control group by every half-year from 2009 to 2018.

Figure 3. Regional recurrence rate among elective and radical neck dissection groups in different periods (before and after October, 2015).

Supplementary figure 1. Mean lymph node yield between target and control groups by every quarter from 2009 to 2018.

\section{Hosted file}

Figures.docx available at https://authorea.com/users/317513/articles/447586-association-oflymph-node-promotion-activity-to-cervical-lymph-node-yield-of-oral-cancer-patients-inasian-country-a-difference-in-differences-analysis

\section{Hosted file}

Tables.docx available at https://authorea.com/users/317513/articles/447586-association-oflymph-node-promotion-activity-to-cervical-lymph-node-yield-of-oral-cancer-patients-inasian-country-a-difference-in-differences-analysis 